\title{
Temperature Dependencies of the Aggregation Properties of RBC in Dextran Solutions In Vitro
}

\author{
Petr B. Ermolinkiy ${ }^{1 *}$, Anastasia I. Maslyanitsina ${ }^{1}$, Andrei E. Lugovtsov ${ }^{\mathbf{1}, 2}$, \\ and Alexander V. Priezzhev ${ }^{1,2}$ \\ ${ }^{1}$ Department of Physics, M.V. Lomonosov Moscow State University, 1 Leninskie Gory, Moscow 119991, Russia \\ ${ }^{2}$ International Laser Center, M.V. Lomonosov Moscow State University, 1 Leninskie Gory, Moscow 119991, Russia \\ *e-mail: peter.ermolinskiy@biomedphotonics.ru
}

\begin{abstract}
The process of reversible spontaneous aggregation of red blood cells (RBC), as well as the process of their shear stress induced disaggregation, both affect hemorheology and blood microcirculation in the human body. The aim of this work is to estimate the influence of temperature on the aggregation properties of RBC suspended in PBS dextran solutions in vitro. Laser method based on diffuse light scattering was used to estimate the RBC aggregation properties. The results demonstrate a clear dependence of the critical shear stress aggregation parameter of RBC in PBS dextran solutions on temperature. These results would help to better understand the process of RBC aggregation. (C) 2020 Journal of Biomedical Photonics \& Engineering.
\end{abstract}

Keywords: RBC aggregation; temperature; erythrocytes; diffuse light scattering; dextran; critical shear stress.

Paper \#3372 received 23 May 2020; revised manuscript received 17 Jun 2020; accepted for publication 18 Jun 2020; published online 25 Jun 2020. doi: 10.18287/JBPE20.06.020501.

\section{Introduction}

Processes of red blood cells (RBC) aggregation and disaggregation strongly influence the viscosity of blood in vessels and have a general impact human health $[1,2]$. Aggregation properties of blood may be changed due many factors: alteration of blood plasma composition, change in blood temperature, injection of different synthetic macromolecules, RBC aging, pathological alterations, and others [2, 3]. The mechanisms of RBC aggregation, as well as the influence of these factors on it, are not fully understood so far. At the present, there exist two main hypotheses explaining the RBC aggregation process: the "Bridging" model and the "Depletion" model $[2,4,5]$. There is still no evidence that would fully confirm or completely refute any one of these models. There exist some suggestions that the RBC aggregation process can be described as a combination of both models [6]. The development of the RBC aggregation models is vital for clearer predicting the changes of the microrheologic properties of blood due to the alterations of concentration of plasma proteins, temperature, etc. The aggregation properties of blood change due to many socially significant diseases [2]. That is why the new knowledge about RBC aggregation process can be used for the development of future clinical applications.

Dextrans are synthetic macromolecules with molecular weights from 3 to $2000 \mathrm{kDa}$ [7]. Dextran solutions are widely used both in clinical practice to adjust viscosity of blood, in the synthesis of drugdelivering nanoparticles [8], and in experimental studies of RBC aggregation [2, 9]. Thermodynamic parameters of polymer solutions (including dextran solutions) are strongly dependent on temperature [10]. Therefore, the temperature should inevitably influence the aggregation properties of $\mathrm{RBC}$ in these solutions. If we consider in vivo conditions, the temperature of the patient's body can alter due to pathologies, and dextran injections can lead to slightly different effects under different temperatures accordingly. The influence of temperature on the processes of RBC aggregation and disaggregation in different solutions, e.g. in dextran solutions, has not been studied in detail so far. However, it is known that in vitro aggregation properties of RBC in autologous plasma are dependent on the temperature $[3,11]$.

The aim of this work is to estimate the influence of temperature on the RBC aggregation properties in 
solutions of dextran of different molecular weights and concentrations in vitro.

\section{Materials and Methods}

The laser aggregometry technique was used to estimate the aggregation properties of $\mathrm{RBC}$ in dextran solutions at different temperatures $[12,13]$. It is based on measuring the intensity of diffuse light scattering from a blood sample and is implemented in the slit-flow type system RheoScan (Rheomeditech, Seoul, Korea) [14]. In more detail, this technique and measured parameters are described in Refs. [3, 13, 15]. Briefly, RheoScan allows to measure kinetics of scattered light and to relate them to the RBC aggregation and disaggregation parameters. One of these parameters is critical shear stress (CSS) that characterizes the shear stress required to balance the process of aggregates formation and aggregates destruction, and therefore CSS is related to the hydrodynamic strength of the RBC aggregates. In this work, only the CSS parameter was measured.

Blood from one healthy individual was drawn using venipuncture technique and was stabilized by EDTA anticoagulant. RBCs were separated from the plasma using standard protocols, then they were suspended and washed in PBS 3 times at $600 \mathrm{~g}$ for $3 \mathrm{~min}$. Washed RBC were suspended in dextran solutions (PBS + dextran) at hct $=0.4$. In this work, dextran macromolecules with different molecular weights $(70,150,500 \mathrm{kDa})$ were used and suspended in PBS at various concentrations (10 $\mathrm{mg} / \mathrm{ml}$ to $100 \mathrm{mg} / \mathrm{ml})$. Prepared samples were incubated for $40 \mathrm{~min}$ at room temperature $\left(22^{\circ} \mathrm{C}\right)$. After that, the blood samples were placed for $5 \mathrm{~min}$ into the RheoScan measurement chamber that was preheated up to 30,37 , or $40{ }^{\circ} \mathrm{C}$ and then measurements were conducted. The temperature of the RheoScan measurement chamber was maintained. Each sample corresponds to one temperature. All experiments were carried out during $5 \mathrm{~h}$ after blood drawing. This time period on its own is not sufficient to cause any significant CSS changes.

\section{Results and Discussion}

Fig. 1 shows the CSS values measured for 3 different temperatures $\left(30,37,40{ }^{\circ} \mathrm{C}\right)$ and for the dextrans of 3 different molecular weights $(70,150$ and $500 \mathrm{kDa})$ and 4 concentrations $(10,30,60$ and $100 \mathrm{mg} / \mathrm{ml})$. The bell shaped dependencies of CSS on dextran concentration are observed in all cases. The graphs shift horizontally at different temperatures. In particular, for dextran $150 \mathrm{kDa}$ (Fig. 1b) the increase in temperature corresponds to shifting to the left, so that the maximum CSS is reached at lower concentration. In the case of dextran $70 \mathrm{kDa}$ and dextran $500 \mathrm{kDa}$ (Fig. 1a and Fig. 1c), the trends are different. The maximum CSS values for 37 and $40{ }^{\circ} \mathrm{C}$ are almost the same and differ from the one at $30{ }^{\circ} \mathrm{C}$. Thus, the molecular weights influence the way how CSS changes under different temperatures. These results mean that there is a nonlinear dependence of CSS parameter on dextran molecular weight, its concentration and the temperatures of the sample. Also, it means that there may be complex influence of combination of these 3 factors (dextran molecular weight, dextran concentration, and temperature) on the RBC aggregation properties.
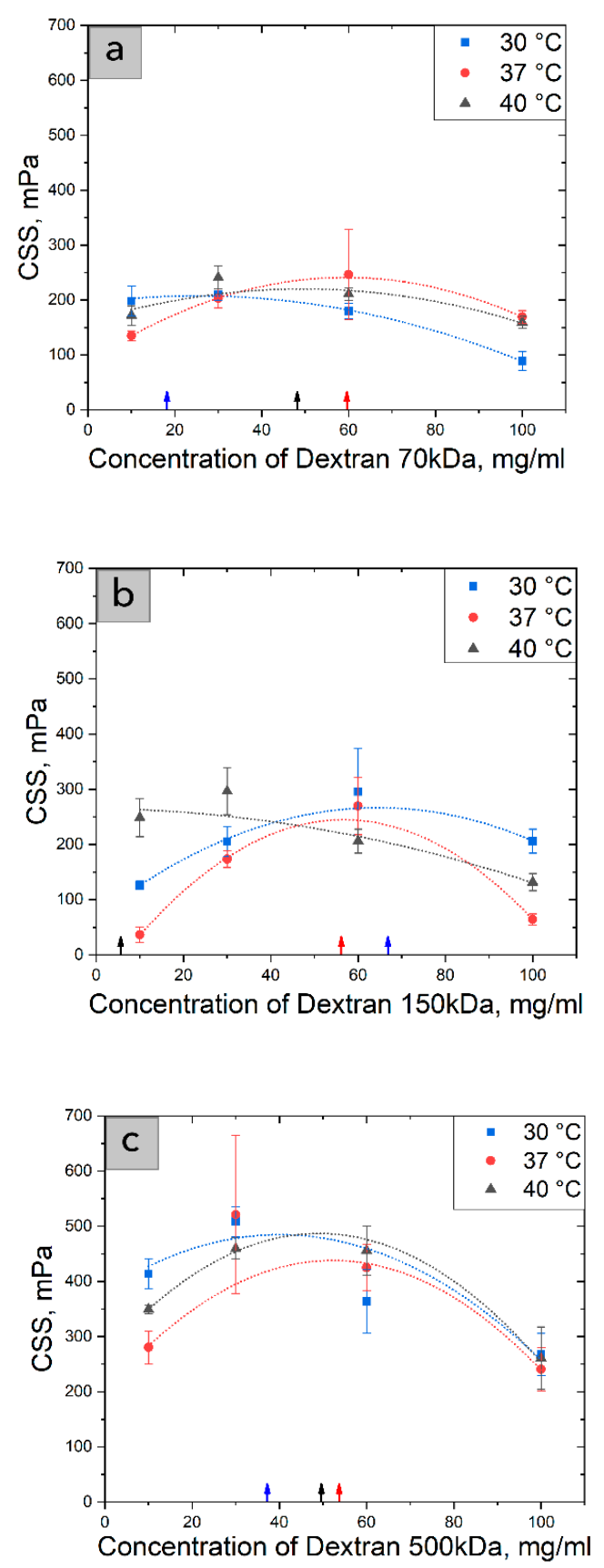

Fig. $1 \mathrm{CSS}$ at different temperatures and concentrations of dextran (a) 70, (b) 150 and (c) $500 \mathrm{kDa}$. Bars represent standard deviations. Each point is an average of at least 5 measurements. Dotted lines are parabolic approximations of experimental data. Colored arrows on horizontal scale correspond to the maximum CSS values. 
Dextran $70 \mathrm{kDa}$ is widely used in clinical practice, namely $6-7.5 \%$ dextran $70 \mathrm{kDa}$ solutions are commonly used for injections to control blood viscosity [16]. Therefore, a more accurate observation of CSS changes was made for dextran $70 \mathrm{kDa}$. Results in Fig. 2 show CSS as a function of temperature for $50 \mathrm{mg} / \mathrm{ml}$ concentration of dextran $70 \mathrm{kDa}$. Interestingly, the minimum CSS values are observed at $30{ }^{\circ} \mathrm{C}$. This also indicates that there is a complex dependence of CSS on temperature.

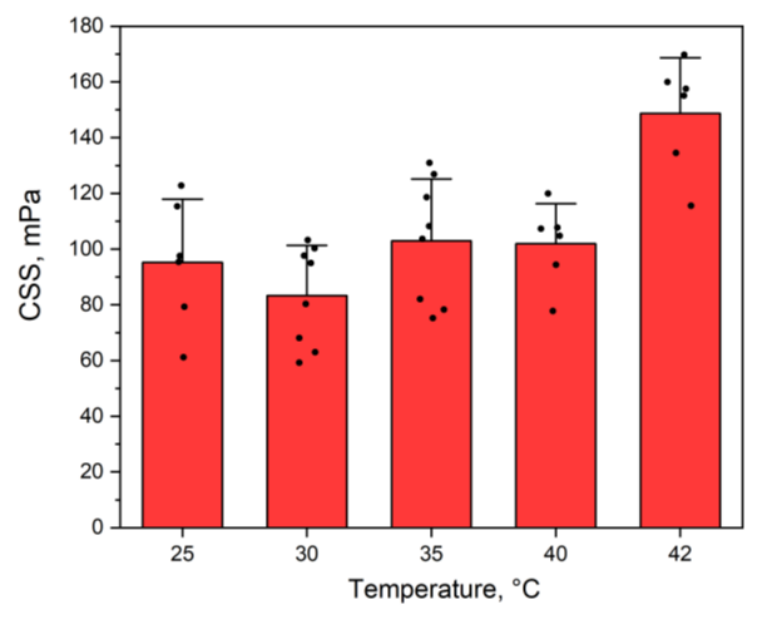

Fig. $2 \mathrm{CSS}$ at different temperatures of dextran $70 \mathrm{kDa}$ solution at concentration of $50 \mathrm{mg} / \mathrm{ml}$. Bars represent standard deviations. Each point corresponds to the one measurement.

In future, it is planned to relate the obtained results with one of the aggregation models. The temperature dependencies of these models are not presented explicitly and need to be developed. The thermodynamic parameters of dextrans depend on temperature, therefore the temperature influences the adsorption of dextran macromolecules on RBC membrane in the case of the "Bridging" model. Also, temperature influences the osmotic pressure in the case of the "Depletion" model. That is why these kinds of results could verify one of the RBC aggregation models in the future.

\section{Conclusion}

In this work, the aggregation of RBC was studied by assessing the critical shear stress (CSS) that characterizes the hydrodynamic strength of the aggregates. The results show a dependence of CSS in dextran solutions on the temperature changes in vitro. In particular, the bell shape dependence of CSS on dextran concentration changes under different temperatures and different molecular weights of dextrans. For example, the concentration of dextran $150 \mathrm{kDa}$ that corresponds to the maximum CSS value is lower for $40^{\circ} \mathrm{C}$ than for $30{ }^{\circ} \mathrm{C}$. These observations mean that there is a nonlinear effect of dextran concentration, dextran molecular weights and temperature on RBC aggregation properties. In future, the fundamental knowledge about RBC aggregation can be used in the development of new methods of patient treatment in order to control the viscosity of blood and rheological parameters.

\section{Disclosures}

All authors declare that there is no conflict of interests in this paper.

\section{Acknowledgments}

This work was supported by the Russian Foundation for Basic Research grant \#19-52-51015.

\section{References}

1. A. N. Yaroslavsky, I. V. Yaroslavsky, "Optics of blood," Chapter 2 in Handbook of Optical Biomedical Diagnostics, V. V. Tuchin (ed.), Volume 1: Light-Tissue Interaction, Second edition, SPIE PRESS, Bellingham, Washington, USA, 161-220 (2016).

2. O. Baskurt, B. Neu, and H. J. Meiselman, Red Blood Cell Aggregation, CRC Press, Boca Raton, USA (2012).

3. P. B. Ermolinkiy, A. N. Semenov, A. E. Lugovtsov, C. Poeschl, U. Windberger, E. Kaliviotis, and A. V. Priezzhev, "Effect of different macromolecules on viscous and microrheologic properties of blood at various temperatures," Proceeding of SPIE 11065, 1106507 (2019).

4. D. E. Brooks, "Mechanism of red cell aggregation," Chapter in Blood Cells, Rheology and Aging, D. Platt (Ed.), Springer, Berlin, Heidelberg, 158-162 (1988).

5. Z. W. Zhang, B. Neu, "Role of macromolecular depletion in red blood cell adhesion," Biophysical Journal 97(4), 1031-1037 (2009).

6. R. Fantoni, A. Giacometti, and A. Santos, "Bridging and depletion mechanisms in colloid-colloid effective interaction: A reentrant phase diagram," The Journal of Chemical Physics 142(22), 224905 (2015).

7. T. Heinze, T. Liebert, B. Heublein, and S. Hornig, "Functional polymers based on dextran," Chapter in Polysaccharides II, D. Klemm (Ed.), Springer-Verlag Berlin Heidelberg, New York, 199-293 (2006).

8. I. Wasiak, A. Kulikowska, M. Janczewska, M. Michalak, I. A. Cymerman, A. Nagalski, P. Kallinger, W. W. Szymanski, and T. Ciach “Dextran nanoparticle synthesis and properties," PLoS ONE 11(1), e0146237 (2016). 
9. N. Antonova, P. Riha, and I. Ivanov, "Experimental evaluation of mechanical and electrical properties of RBC suspensions under flow. Role of RBC aggregating agent," Clinical Hemorheology and Microcirculation 45(2-4), 253-261 (2010).

10. A. Gunner, G. Kibarer, "The important role of thermodynamic interaction parameter in the determination of theta temperature, dextran/water system," European Polymer Journal 37(3), 619-622 (2001).

11. M. Singh, J. F. Stoltz, "Influence of temperature variation from $5^{\circ} \mathrm{C}$ to $37^{\circ} \mathrm{C}$ an aggregation and deformability of erythrocytes," Clinical hemorheology and microcirculation 26(1), 1-7 (2002).

12. A. V. Priezzhev, K. Lee, N. N. Firsov, and J. Lademann, "Optical study of RBC aggregation in whole blood samples and single cells," Chapter in Handbook of Optical Biomedical Diagnostics, V. V. Tuchin (Ed.), Volume 2: Methods, Second edition, SPIE PRESS, Bellingham, Washington, USA, 5-36 (2016).

13. S. Shin, J. H. Nam, J. X. Hou, and J. S. Suh, “A transient, microfluidic approach to the investigation of erythrocyte aggregation: The threshold shear-stress for erythrocyte disaggregation," Clinical Hemorheology and Microcirculation 42(2), 117-125 (2009).

14. V. N. Lopatin, A. V. Priezzhev, and A. D. Aponasenko, Methods light scattering in the analysis of dispersed biological media, Fizmatlit, Moscow, Russia (2004) [in Russian]. ISBN 5-9221-0547-7.

15. A. N. Semenov, A. E. Lugovtsov, K. Li, A. A. Fabrichnova, Y. A. Kovaleva, and A. V. Priezzhev, “Applying methods of diffuse light scattering and optical trapping for assessing blood rheological parameters: erythrocytes aggregation in diabetes mellitus," Izvestiya of Saratov University. New series. Series Physics 17(2), 85-97 (2017) [in Russian].

16. A. Gray, J. Wright, V. Goodey, and L. Bruce, Injectable drugs guide, Pharmaceutical Press. Royal pharmaceutical society, London, UK (2011). 